\title{
Mars Conjunction Crewed Missions with a Reusable Hybrid Architecture
}

\author{
Raymond G. Merrill \\ NASA Langley Research \\ Center \\ 1 North Dryden Street MS 462 \\ Hampton, VA 23681 \\ 757-864-2762 \\ Raymond.G.Merrill@nasa.gov
}

\author{
Nathan Strange \\ Jet Propulsion Laboratory, \\ California Institute of \\ Technology \\ 4800 Oak Grove Dr. \\ Pasadena, CA 91109 \\ 818.393-1165 \\ Nathan.J.Strange@nasa.gov
}

\author{
Min Qu \\ AMA, Inc. \\ 21 Enterprise \\ Parkway Suite 300 \\ Hampton, VA \\ 23666 \\ 757-864-9737 \\ M.Qu-1@nasa.gov
}

\author{
Noble Hatten \\ The University of Texas at \\ Austin \\ Department of Aerospace \\ Engineering and Engineering \\ Mechanics \\ 1 University Station, C0600 \\ Austin, TX 78712 \\ 512-657-2450 \\ Noble.Hatten@gmail.com
}

\begin{abstract}
A new crew Mars architecture has been developed that provides many potential benefits for NASA-led human Mars moons and surface missions beginning in the 2030s or 2040s. By using both chemical and electric propulsion systems where they are most beneficial and maintaining as much orbital energy as possible, the Hybrid spaceship that carries crew round trip to Mars is pre-integrated before launch and can be delivered to orbit by a single launch. After check-out on the way to cis-lunar space, it is refueled and can travel round trip to Mars in less than 1100 days, with a minimum of 300 days in Mars vicinity (opportunity dependent). The entire spaceship is recaptured into cis-lunar space and can be reused. The spaceship consists of a habitat for $\mathbf{4} \mathrm{crew}$ attached to the Hybrid propulsion stage which uses long duration electric and chemical in-space propulsion technologies that are in use today. The hybrid architecture's con-ops has no in-space assembly of the crew transfer vehicle and requires only rendezvous of crew in a highly elliptical Earth orbit for arrival at and departure from the spaceship. The crew transfer vehicle does not travel to Mars so it only needs be able to last in space for weeks and re-enter at lunar velocities. The spaceship can be refueled and resupplied for multiple trips to Mars (every other opportunity). The hybrid propulsion stage for crewed transits can also be utilized for cargo delivery to Mars every other opportunity in a reusable manner to pre-deploy infrastructure required for Mars vicinity operations. Finally, the Hybrid architecture provides evolution options for mitigating key long-duration space exploration risks, including crew microgravity and radiation exposure.
\end{abstract}

Table of Contents

1. INTRODUCTION ........................................1

2. A HYBRID ARCHITECTURE ........................2

3. FLIGHT ELEMENTS..................................3

4. CREW MISSION DESIGN ................................5

5. CARGO MISSION DESIGN ...............................

6. SENSITIVITY ANALYSES .................................8

7. EVOLVABILITY .............................................11

8. CONCLUSIONS................................................11

ACKNOWLEDGMENTS...............................................11

REFERENCES...........................................................11

BIOGRAPHY ............................................................12

\section{INTRODUCTION}

The Evolvable Mars Campaign (EMC) is NASA's next iteration on a Human Mars exploration plan. The lessons learned over the past 40 years of government led space exploration are applied through "six strategic principals to provide a sustainable program:

1. Implementable in the near-term with the buying power of current budgets and in the longer term with budgets commensurate with economic growth.

2. Application of high Technology Readiness Level (TRL) technologies for near term, while focusing research on technologies to address challenges of future missions

3. Near-term mission opportunities with a defined cadence of compelling missions providing for an incremental buildup of capabilities for more complex missions over time

4. Opportunities for US Commercial Business to further enhance the experience and business base learned from the ISS logistics and crew market

5. Multi-use, evolvable Space Infrastructure

6. Significant International and Commercial participation, leveraging current International Space Station partnerships.”[1]

The EMC starts with NASA as it is today. Humans are in space continuously at the International Space Station (ISS). NASA's Human Exploration and Operations Mission Directorate (HEOMD) must maintain and operate the ISS while pursuing Human spaceflight in-space capability development for future missions. Infrastructure and operations costs must be lowered for NASA to expand capabilities with near term buying power. NASA must move from operating a logistics infrastructure for a location 400 miles away from Earth's surface to operating one that services multiple assets hundreds of thousands of miles to millions of miles from Earth's surface. 
In order to field any human Mars mission a set of at least 5 capabilities must be developed into future spacecraft. These capabilities are:

1. Crew and cargo access to space

2. Long duration beyond Earth crew support

3. Long duration in-space transportation

4. Destination access

5. Destination systems

This list can be shortened to only the first three capabilities for initial missions defined in the Flexible Path for exploration [2] through Mars orbit access and the exploration of Phobos and Deimos. This allows phasing of Mars surface systems while continuing a cadence of compelling missions.

Capability 1 exists for access to Low Earth Orbit (LEO), however access to cis-lunar space near the edge of Earth's sphere of influence provides an aggregation and departure location that reduces the Earth Departure stage size dramatically and allows for inexpensive recapture of systems from heliocentric space. NASA is developing the Space Launch System (SLS) and Orion vehicles for this purpose. Commercial or International ISS access vehicles also represent capabilities that could be extended to enable cislunar crew and cargo capabilities.

Several potential cis-lunar orbits have been identified including some that are long-term stable while others are loosely bound and require constant upkeep. These orbits are the next logical destination for long term Human presence. They are all very similar in orbital energy and a spacecraft can maneuver between them for small velocity increments $(\Delta \mathrm{V})$ given enough time. Transfers from Earth to these orbits can be accomplished quickly for crew access. Cis-lunar space is also the easiest location to reach to beyond the Van-Allen belts and the micrometeoroid and orbital debris environment around Earth. A ballet of ballistic transfers leveraging solar perturbations [3] and Lunar Gravity effects [4] far from the Earth can now be leveraged to perform transfers and Earth departures or captures. Even very large masses, such as small asteroids can be captured from near Earth Space with very low $\Delta \mathrm{V}$ and small amounts of force applied over long periods of time.[5]

Capability 2, long duration beyond Earth crew support systems, can be tested in a location where the Earth and Moons gravity interacts. The concept of an outpost or Cislunar Base Camp [6] can be used to develop the capabilities and the confidence in them required to extend human presence beyond Earth into the inner solar system. Trips for crew to and from cis-lunar space are on the order of ten to twelve days and allow for abort to Earth in the event of a critical system failure.

Finally capability 3 , long duration in-space transportation systems, provide mobility for crew and destination systems. Initially crew elements are transported to and maintained in cis-lunar space. The in-space propulsion system can facilitate transits around Earth - Moon and Sun - Earth Space for "sea- trials" and shake downs. The Orion chemical propulsion system is long duration storable, re-startable, and has heritage with the Space Shuttle Orbital Maneuvering System (OMS). The Asteroid Redirect Robotic Mission is developing Solar Electric Propulsion (SEP) capabilities and magnetically shielded Hall Thrusters in the tens of kilowatt range that will be rated for many years of service by the end of the mission. It is possible to use a combination of Chemical Propulsion (CP) and Electric Propulsion (EP) at these performance points to enable transportation of humans to Mars orbit. Using these existing investments and capabilities, NASA can minimize the uncertainty and improve the sustainability of an inner solar system human presence.

This paper presents a hybrid architecture, the EMC element concepts being developed by NASA's Human exploration Architecture Team (HAT) and the resulting mission design for transit of crew and cargo to Mars.

\section{A Hybrid ARCHitecture}

Only a few combined low thrust and high thrust Human missions have been proposed recently. Missions to NEAs and Mars previously developed by the authors $[7,8]$ as part of the Electric Path [9] only used the CP component of the mission for Earth departure from a high elliptical orbit. Conversely the Mars mission described by Mercer et al. [10] only uses the CP component for Mars orbit arrival and departure from an elliptical 1-sol Mars orbit.

This new Hybrid architecture includes three key strategies that guide mission design decisions.

- Use celestial energy resources to save propellant where time allows

- Maintain maximum orbital energy for the crew transport spaceship

- Re-use in-space architecture elements as much as possible

These strategies are in the design a crew architecture that is comparable to a conjunction class Mars mission $(<1100$ day total duration $>300$ day Mars vicinity), but can be accomplished without staging any component of the vehicle or need to rendezvous with additional elements or fuel. The Hybrid Mars crewed round trip mission can be described by a set of phases (Figure 1).

A common theme for human deep space missions is aggregation and assembly of propulsion and crew support elements. The ability to launch an entire spaceship that is pre-integrated and able to fly round trip to Mars has only been possible with very large launch vehicles. One of the Hybrid architecture's objectives is to enable launch of an integrated vehicle that only needs to be re-fueled and maintained in order to enable multiple trips from cis-lunar space to Mars. Each hybrid propulsion stage can enable one conjunction class trip to Mars every other opportunity (4 2/7 years). 
In order to minimize the spaceship size, orbital energy is maximized and propellant required is minimized across trajectories from cis-lunar space to and from Mars by utilizing a combination of Lunar Gravity Assists (LGA), solar perturbation loops, and high energy elliptical parking orbits. Earth departure is via a LGA from a high elliptical orbit. Crew rendezvous with the Mars spaceship in the high elliptical orbit to enable quick propellant-less Earth departure. The crew return to Earth and rendezvous with their return capsule using the same maneuvers performed in reverse. EP is used during the transit to and from Mars, where a high energy elliptical parking orbit is used with period between 5 and 10 sol.

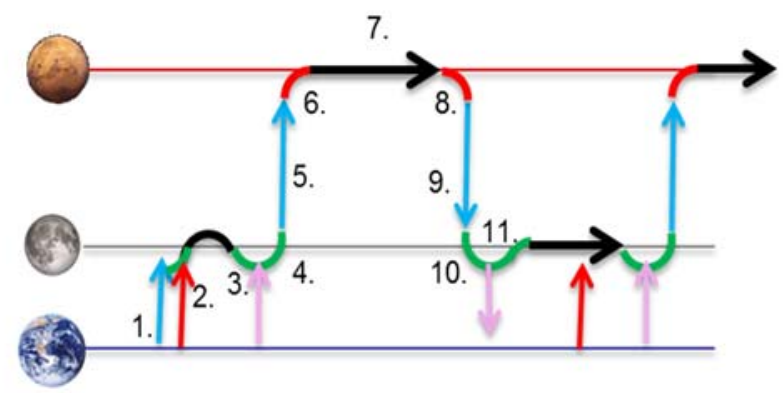

1. Deploy to cis-lunar space

2. Refuel and outfit

3. Crew and final logistics rendezvous

4. Powered Lunar Gravity Assist (LGA) for Earth departure

5. Solar Electric Propulsion (SEP) thrusting to Mars

6. Chemical Propulsion (CP) burn at Mars close approach to insert into parking orbit

7. Mars destination operations

8. CP burn and periapsis for Mars departure

9. SEP thrusting to Earth

10. LGA for Earth capture into cis-lunar space

11. Crew return to Earth surface

Repeat steps 2-11 for each crew Mars mission

Figure 1. Hybrid Architecture Mission Phases

In order to venture in space for years at a time we must eventually mitigate long duration effects of leaving Earth's surface. Crew support hardware that mitigates radiation and micro-gravity effects on humans is more massive than the system described in Section 3. The Hybrid architecture provides evolution paths for much heavier vehicles at similar power and thrust levels through a ballistic cycler architecture so that future pioneers need not be exposed to the same detrimental deep space environmental influences that initial explorers endure.

\section{Flight Elements}

\section{Launch Vehicles}

SLS Block 2: Delivers crew or cargo to Cis-lunar locations including Lunar Distant Retrograde Orbit (LDRO) and Lunar
Distance High Earth Orbit (LDHEO). Also delivers larger cargo up to $75 \mathrm{t}$ to lower elliptical orbits. [11]

Commercial launch vehicle fleet including Delta IV Heavy and Falcon Heavy: Delivers cargo to LDRO or LDHEO

\section{Crew Launch and Earth Entry Vehicle}

Orion: Supports crew transit to and return from LDRO or LDHEO on fast transits of no more than 12 days one way.

\section{Long Duration Crew Support Systems}

Deep Space Habitat: $40 \mathrm{t}$ fully outfitted and has a dry mass of $22 \mathrm{t}$ without logistics or spares and supports a crew of 4 for up to 1100 days in deep space. $32 \mathrm{t}$ fully outfitted for a crew of 4 for 600 days. [12]

\section{Phobos and Deimos Exploration and Crew Support}

Habitat and Exploration Equipment: Supports for multiple Phobos stays (approximately 500 days total duration with delivered logistics). Including exploration vehicles and equipment to enable science on Phobos and Deimos. [13]

\section{In-space Transportation}

Hybrid Propulsion Stage: Element is a combination of Solar Electric Propulsion (EP) and Chemical Propulsion (CP). Parametrically sized at $212 \mathrm{~kW}$ electric propulsion power with high thrust and high Specific impulse ( $\mathrm{I}_{\mathrm{SP}}$ ) modes ( $\sim 2000 \mathrm{~s}$ and $\sim 3000 \mathrm{~s}), 400 \mathrm{~kW}$ array at $1 \mathrm{AU}, 16 \mathrm{t}$ xenon capacity, and $16 \mathrm{t}$ bi-prop capacity with $\mathrm{I}_{\mathrm{SP}}$ of $319 \mathrm{~s}$. Stage inert mass is estimated at $15.1 \mathrm{t}$ with a wet mass of $48 \mathrm{t}$. Details of the stage are listed in Figure 2.

\section{Mars Sphere of Influence (SOI) Crew Transport}

Mars Taxi transports crew from Mars parking orbits to Phobos. Can support a crew of 4 for up to 7 days and has a bi-prop propulsion stage that is $1 / 2$ the Hybrid vehicle CP with $8 \mathrm{t}$ propellant.

\section{Mars Surface Access}

Mars Landers: pre-deploys the required surface assets and ascent stage. Currently two designs are being evaluated, one is $59 \mathrm{t}$ at Mars arrival and delivers $26 \mathrm{t}$ of payload and the other is $43 \mathrm{t}$ at Mars arrival and delivers $18 \mathrm{t}$ of payload to the surface. 


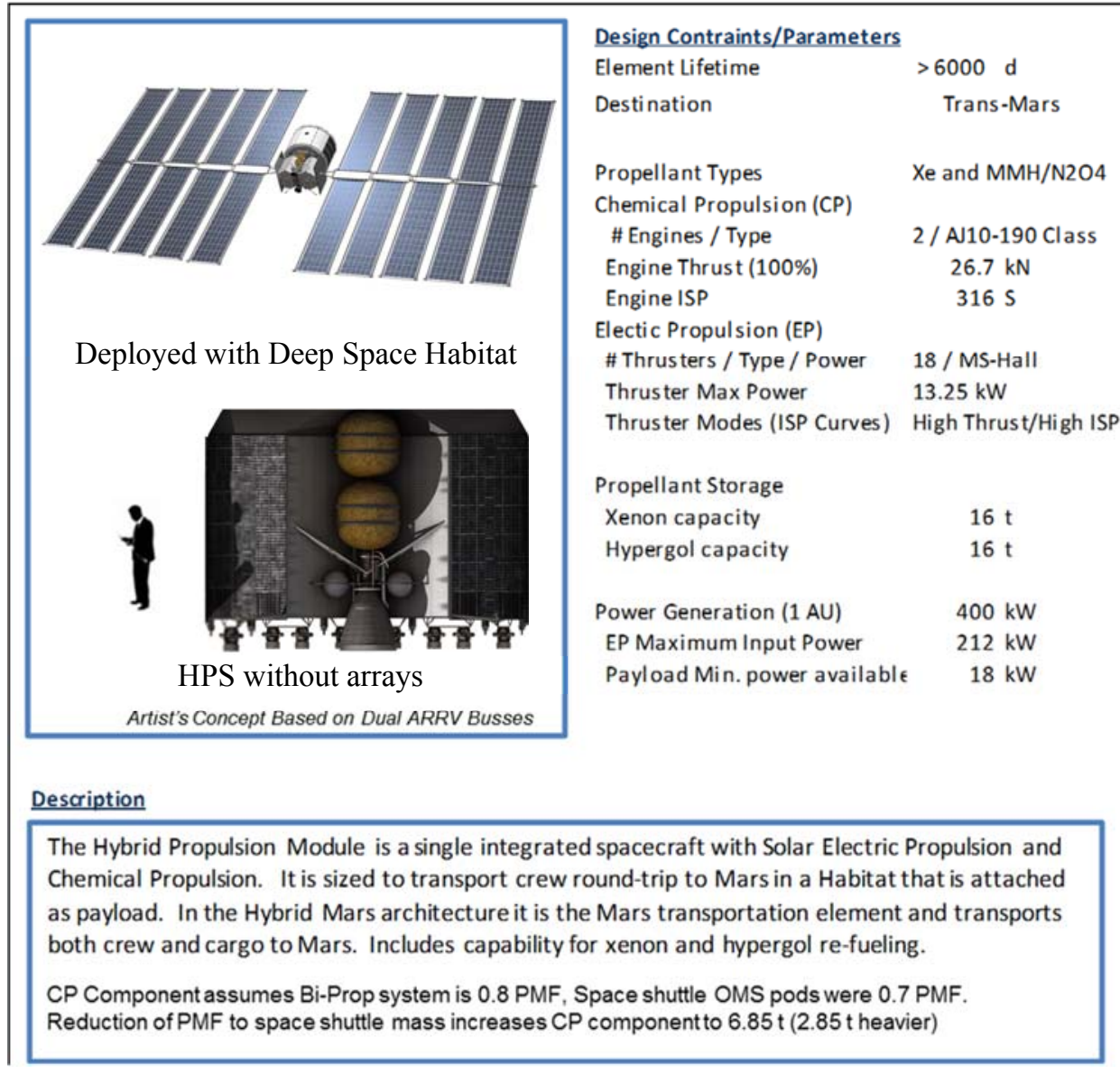

\begin{tabular}{|l|c|}
\hline Category & Mass, kg \\
\hline Array + EP Component & 11103 \\
\hline CP Component & 4000 \\
\hline & \\
\hline & \\
\hline & \\
\hline & \\
\hline & \\
\hline & \\
\hline DRY MASS SUBTOTAL & 15103 \\
\hline INERT MASS SUBTOTAL & 15103 \\
\hline Non-propellant & \\
\hline RCS Propellant & 800 \\
\hline Bi-Prop Propellant and Oxidizer & 16000 \\
\hline Xenon & 16000 \\
\hline TOTAL WET MASS & 47903 \\
\hline
\end{tabular}
Array + EP and RCS Component Parametric
Mass $(\mathrm{kg})=2274.61+30.1925 *$ EP Power $(\mathrm{kW})$ $+0.0552 *$ Propellant $(\mathrm{kg})$ $+8 *$ (Array Power - EP Power) $(\mathrm{kW})$ CPS Component Parametric

Dry Mass $(\mathrm{kg})=$ Fuel $*(1 / 0.8)-$ Fuel

Hybrid Architecture Mars Payloads:

Crew Habitat with Logistics $\quad 40,000 \mathrm{~kg}$

Round Trip $<1100 \mathrm{~d}$ with Stay $>300 \mathrm{~d}$

Phobos Habitat and Taxi $\quad 40,495 \mathrm{~kg}$

Outbound to Phobos, with taxi drop at SOI

27t PL Mars Lander $59,000 \mathrm{~kg}$

Dropped at SOI, returns to Earth for re-use

Figure 2. Hybrid Propulsion Stage Concept

\section{Mars}

Phobos

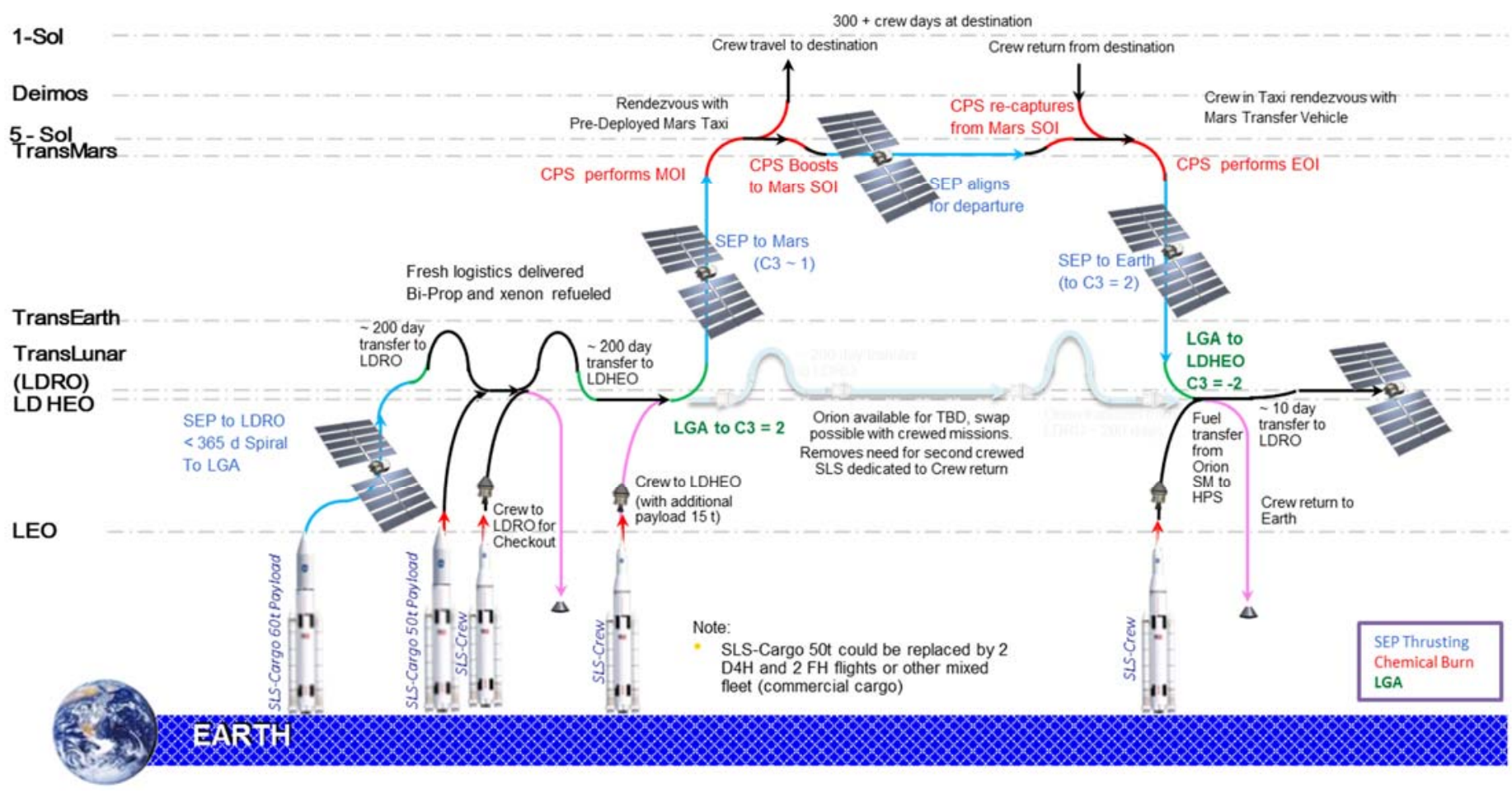

Figure 3. Hybrid EMC architecture Bat chart (crew portion). 


\section{CReW Mission Design}

The initial Hybrid crew mission is depicted in Figure 3. Additional crew missions that re-use the integrated Mars spaceship begin with the vehicle in LDRO after the previous use.

\section{Deployment to LDRO}

The crewed Mars mission begins with initial deployment and checkout of the integrated Hybrid Propulsion Stage (HPS) and habitat stack. They are launched by a SLS 2 to an elliptical orbit $(200 \mathrm{~km} \times 20,000 \mathrm{~km})$ where the SEP is deployed and thrusts for approximately one year to reach a Lunar Gravity Assist (LGA), from there the trip to stable cislunar space takes approximately 6 months. Upon arrival in a LDRO and rendezvous with the existing infrastructure additional SLS is launched to LDRO with all the fuel and additional logistics required for the Mars mission. A crew can visit the Mars spaceship and perform habitat checkout and outfitting if necessary. The advantage of the LDRO in that it is stable, other locations in cis-lunar space must be maintained via small maneuvers weekly or bi-weekly.

\section{Fast Transits to LDRO}

Crew transfers to and from the LDRO have been developed with 10-12 day transits from Earth for the Asteroid Redirect Crewed Mission (ARCM) [14]. These transits include 3 maneuvers to leave the LDRO, an initial departure of $\sim 20$ $\mathrm{m} / \mathrm{s}$, a targeting maneuver approximately 3 days later to target a lunar close approach of $\sim 110 \mathrm{~m} / \mathrm{s}$ and a powered lunar swing-by approximately 4 days later of $\sim 180 \mathrm{~m} / \mathrm{s}$. The total $\Delta \mathrm{V}$ for a crew arriving or departing is $\sim 310 \mathrm{~m} / \mathrm{s}$. If an all chemical Mars transit vehicle uses these maneuvers before Trans Mars Injection (TMI) between 470 and $900 \mathrm{~m} / \mathrm{s} \Delta \mathrm{V}$ is required at Earth close approach to achieve the necessary hyperbolic excess velocity for a trip to mars, dependent on opportunity.

\section{LGA from LDHEO after $L D R O$}

The fast transit described is used to help minimize crew duration in space if the crew rendezvoused with the Mars spaceship in the LDRO and used a CP departure like other architectures that are aggregated in cis-lunar space. There is another class of transfer from an LDRO to LDHEO that is < $70 \mathrm{~m} / \mathrm{s} \Delta \mathrm{V}$ however it takes nearly 6 months to complete the transfer in a manner similar to the Hybrid Spaceship deployment to LDRO. After departure from the LDRO the Mars spaceship transfers to LDHEO via a solar perturbation loop and a pair of LGAs (Fig. 4). Mars crew rendezvous with the Mars spaceship in LDHEO after these maneuvers so they don't have to be onboard during the long transit from LDRO to LDHEO. From LDHEO a LGA propels the Mars spaceship with crew onboard to a characteristic energy (C3) of $2 \mathrm{~km}^{2} / \mathrm{s}^{2}$. The total Earth departure $\Delta \mathrm{V}$ for Hybrid is $<70$ $\mathrm{m} / \mathrm{s}$ and is accomplished at $3000 \mathrm{~s} \mathrm{I}_{\mathrm{SP}}$ by the EP system. If a higher $\mathrm{C} 3$ is required a powered LGA or ECA that use the HPS CP system can be used.

\section{EP to Mars and CP for capture}

After Earth departure the EP system thrusts for much of the outbound trajectory to increase the vehicle's orbital energy to nearly that of Mars. The Mars spaceship arrives at Mars with a low $\mathrm{C} 3$ of between 1 and $2 \mathrm{~km}^{2} / \mathrm{s}^{2}$ with the incoming hyperbola targeted for a Mars close approach at $150 \mathrm{~km}$ altitude. The CP component of the HPS performs the Mars orbit insertion into a high elliptical Mars orbit with period of 5-10 Sol. Using larger Mars orbits reduces the propulsion requirement by more than $50 \%$ compared to 1 Sol orbits for these arrival velocities.

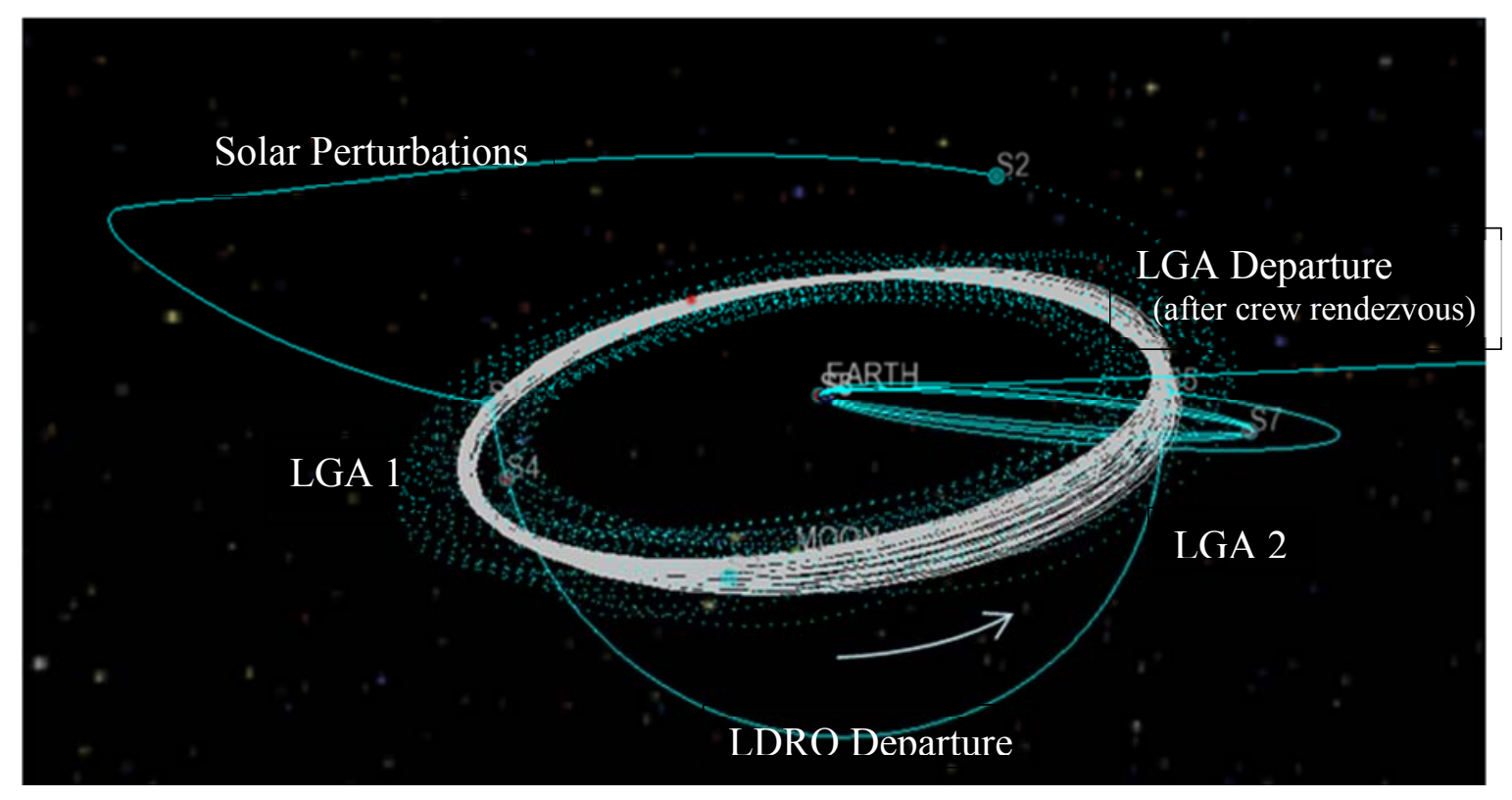

Figure 4. LDRO to LDHEO transfer 


\section{Crew transfers to and from Phobos}

Upon arrival at Mars the pre-deployed taxi rendezvous with the Mars spaceship to transport the crew to Phobos. The transfer is approximately 3 days for a 5-sol orbit and 6 days for a 10 -sol orbit. Depending on the arrival orbit inclination relative to Phobos larger orbits can be less $\Delta \mathrm{V}$ than a transfer from a smaller orbit like a 1 -sol. Figure 5 is the round trip $\Delta \mathrm{V}$ to Phobos for varied total round trip plane change. Ascent from the surface of Mars is also a concern for larger orbits, however an addition of $0.18 \mathrm{~km} / \mathrm{s}$ to a total ascent $\Delta \mathrm{V}$ of more than $5 \mathrm{~km} / \mathrm{s}$ is small. A greater concern to be assessed in the future is the risk posture for a crew ascent to a multi-day period orbit when the ascent module only supports one day of life support nominally.

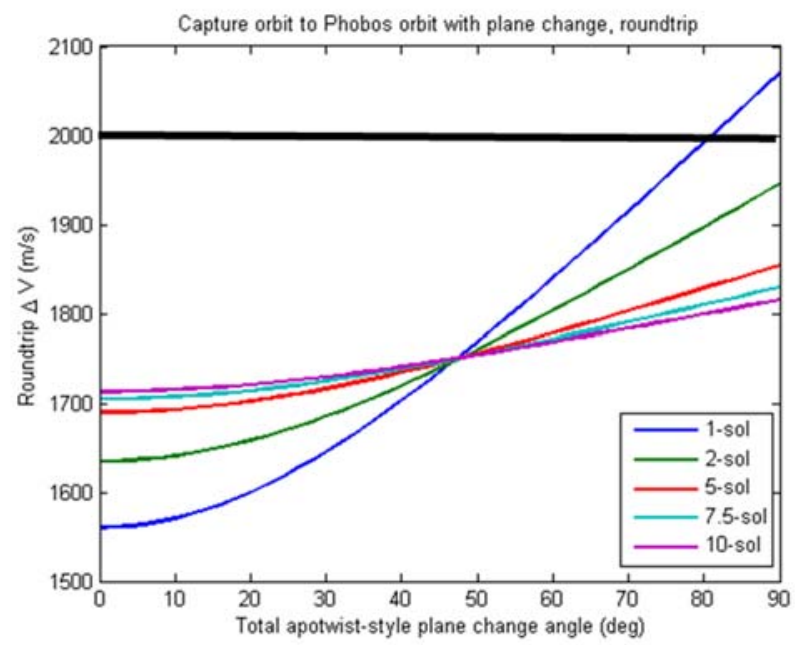

Figure 5: Crew taxi $\Delta$ V for Mars elliptical orbit to Phobos round trip. Horizontal black line represents current split transportation architecture taxi $\Delta \mathrm{V}$ budget.

\section{Mars orbit reorientation}

While the larger Mars parking orbits reduce propulsion requirements for insertion and departure they also pose a challenge for reorientation from arrival orbit to departure orbit. Larger orbits (5-10 sol) do not process as quickly as smaller orbits ( 1 sol). That means it is less likely that a plane change maneuver can be used to re-orient the orbit as the arrival and departure orbits cross. For the Hybrid mission design a set of maneuvers termed the "Butterfly" (Figure 6) have been developed that use third body effects and SEP thrusting near the edge of Mars sphere of influence for alignment. The CP system provides $\sim 35 \mathrm{~m} / \mathrm{s} \Delta \mathrm{V}$ for 10 sol parking orbits or $\sim 65 \mathrm{~m} / \mathrm{s}$ for 5 sol parking orbits to reach the Mars sphere of influence where small

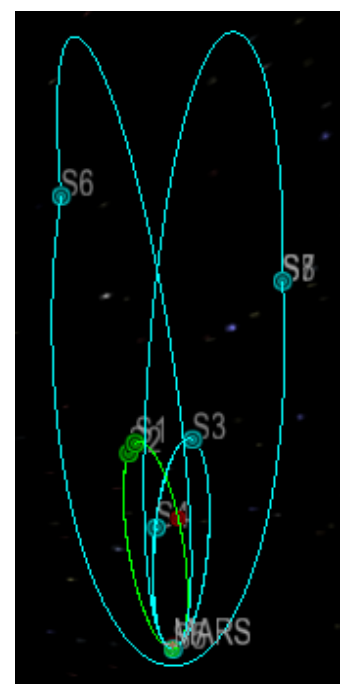

Figure 6. 2037 Butterfly maneuvers reorient the orbit. The same $\Delta \mathrm{V}$ is required for recapture into the departure parking orbit. The 10 Sol transfer in Figure 7 is $110 \mathrm{~m} / \mathrm{s}$ total $\Delta \mathrm{V}$ and 100 days for the transfer. Other opportunities have also been assessed to verify that the crew vehicle can re-orient in time for departure. The duration of the butterfly maneuver varies and is up to 250 days, however the required $\Delta \mathrm{V}$ is nearly the same.

\section{CP Mars departure and EP to LGA}

After a stay in the Mars sphere of influence of at least 300 days the CP component of the HPS performs a burn at perigee to boost the Mars spaceship to a C3 of approximately 1 $\mathrm{km}^{2} / \mathrm{s}^{2}$. From there the EP component uses its high $\mathrm{I}_{\mathrm{SP}}$ mode to transfer the vehicle back to Earth targeting an LGA with an incoming $\mathrm{C} 3$ of $<2 \mathrm{~km}^{2} / \mathrm{s}^{2}$.

\section{Earth Arrival and Crew return to Earth}

The crewed Mars spaceship captures back into a LDHEO via a LGA in a manner similar to Earth departure, but in reverse. An SLS 2 launches Orion to rendezvous with the Mars spaceship and transfers fuel and logistics carried to the HPS before returning the crew to Earth.

\section{Transfer to $L D R O$}

The Mars Spaceship transfers back to the LDRO after the crew depart. Depending on Mars opportunity this transfer may be sped up by using the propellant carried on the crew rendezvous to ensure adequate time in the LDRO for refurbishment and refueling prior to the next Earth departure window to Mars.

Table 1 is crewed durations for Mars Hybrid Missions. Note for the 2033 opportunity the Mars stay is 432 days and for the 2037 opportunity the lower limit of 300 days in the Mars

\section{Table 1. Mars Hybrid Mission Dates}

$\begin{array}{cccccc}\begin{array}{c}\text { Mission } \\ \text { Date }\end{array} & \begin{array}{c}\text { Earth to } \\ \text { Mars }\end{array} & \begin{array}{c}\text { Mars } \\ \text { Stay }\end{array} & \begin{array}{c}\text { Mars to } \\ \text { Earth }\end{array} & \begin{array}{c}\text { Transit } \\ \text { Duration }\end{array} & \begin{array}{c}\text { Total } \\ \text { Duration }\end{array} \\ \text { 3/8/2033 } & 277 & 443 & 308 & 585 & 1028 \\ 7 / 17 / 2037 & 385 & 300 & 350 & 735 & 1035 \\ 9 / 10 / 2041 & 375 & 305 & 380 & 755 & 1060\end{array}$

system is reached. Table 2 is the total propellant and logistics needs for these flights. The logistics can be delivered with the crew on cis-lunar missions and when rendezvousing with the Mars spaceship in LDHEO. Xenon and bi-prop can be launched to LDRO by an SLS 2 in a set of HPS tanks or by commercially contracted fuel delivery.

Table 2. Mars Hybrid Mission Needs

\begin{tabular}{|c|c|c|c|c|c|}
\hline $\begin{array}{c}\text { Mission } \\
\text { Date }\end{array}$ & $\begin{array}{c}\text { Logistics } \\
\text { (t) }\end{array}$ & $\begin{array}{c}\text { Spares } \\
\text { (t) }\end{array}$ & $\begin{array}{c}\text { Xenon } \\
(t)\end{array}$ & $\begin{array}{c}\text { Bi-Prop } \\
\text { (t) }\end{array}$ & $\begin{array}{c}\text { Total } \\
\text { (t) }\end{array}$ \\
\hline $3 / 8 / 2033$ & 14 & 4 & 14 & 14 & 46 \\
\hline $7 / 17 / 2037$ & 14 & 4 & 15 & 15 & 48 \\
\hline 9/10/2014 & 14 & 4 & 16 & 16 & 50 \\
\hline
\end{tabular}




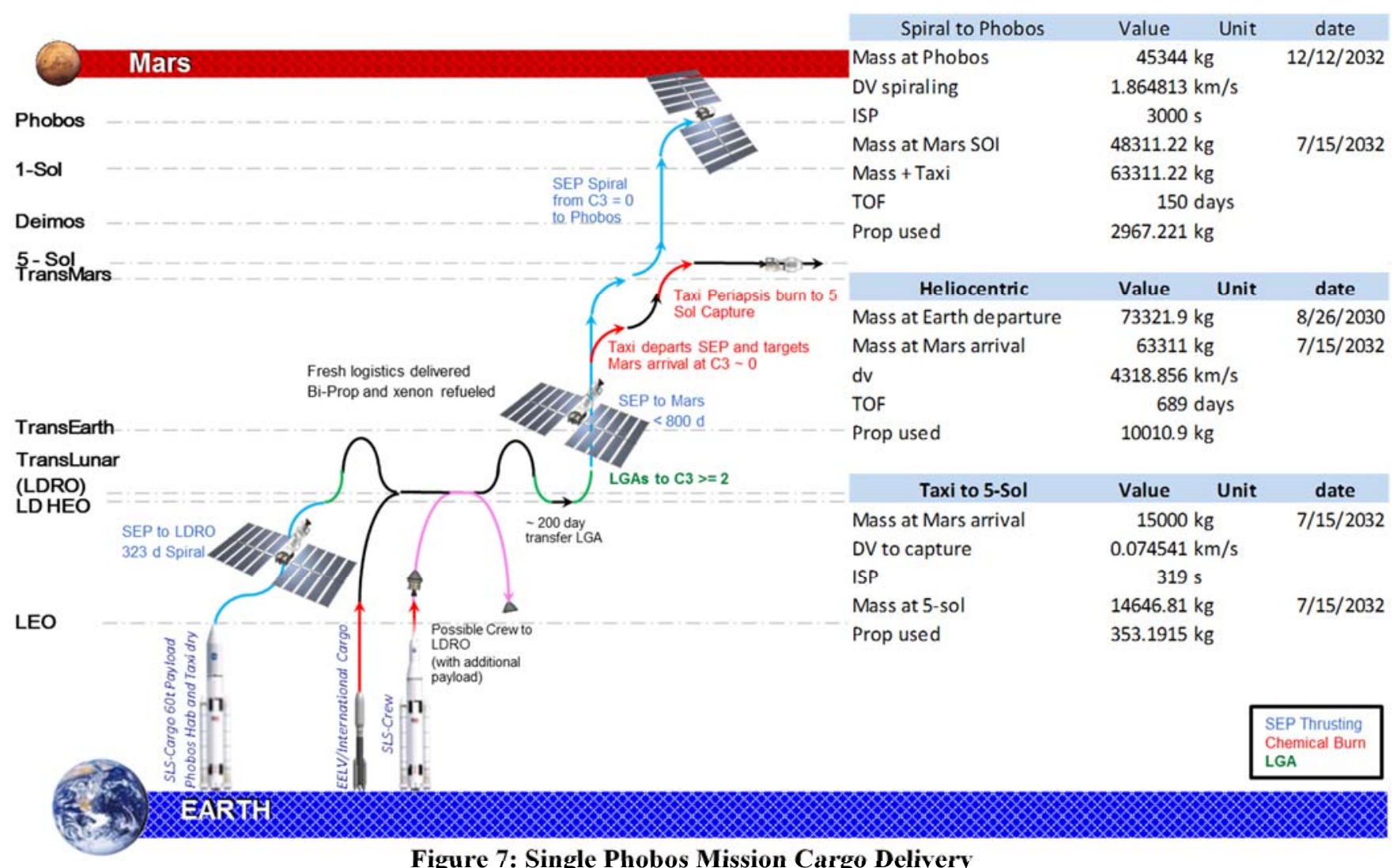

\section{Cargo Mission Design}

The HPS designed for the crewed mission is also used for cargo missions in the Hybrid architecture. The cargo missions in this section are initial feasible options to prove HPS viability for Mars cargo delivery.

\section{Phobos Cargo Delivery}

Phobos cargo delivery capability is limited by the Earth departure mass up to nearly $90 \mathrm{t}$. Two example cargo trajectories were developed. The first is for a single Phobos visit in 2033 (Figure 7) and the second delivers enough logistics and propellant for two round trips from a parking orbit to Phobos. The Crew taxi is delivered to Mars on the Phobos cargo flight, it detaches from the Phobos cargo stack before entering Mars sphere of influence and captures into a high elliptical phasing orbit while the HPS and Phobos habitat spiral to Phobos. A comparison of the usable Phobos payload and the amount of propellant required to refuel the Phobos HPS is illustrated in Figure 8. There is nearly a 1 to 1 relationship between additional mass needed in LDRO for earth departure. This mass can be logistics, fuel, or additional elements to be delivered to Phobos. Mass delivered to 5 sol in this graph is the Phobos taxi and is considered fixed with the available payload at Phobos falling out. It should also be noted that the CP component of the HPS is not used in the 0 $-18 \mathrm{t}$ additional mass needed cases, but is halfway fueled for the $33 \mathrm{t}$ additional mass case to provide propellant for a second round trip. If the $\mathrm{CP}$ component is removed from the HPS for the smaller payload cases $4 \mathrm{t}$ of unusable mass at Phobos could transition to usable mass. Additional use of the
CP component for cargo delivery is an area for additional assessment.

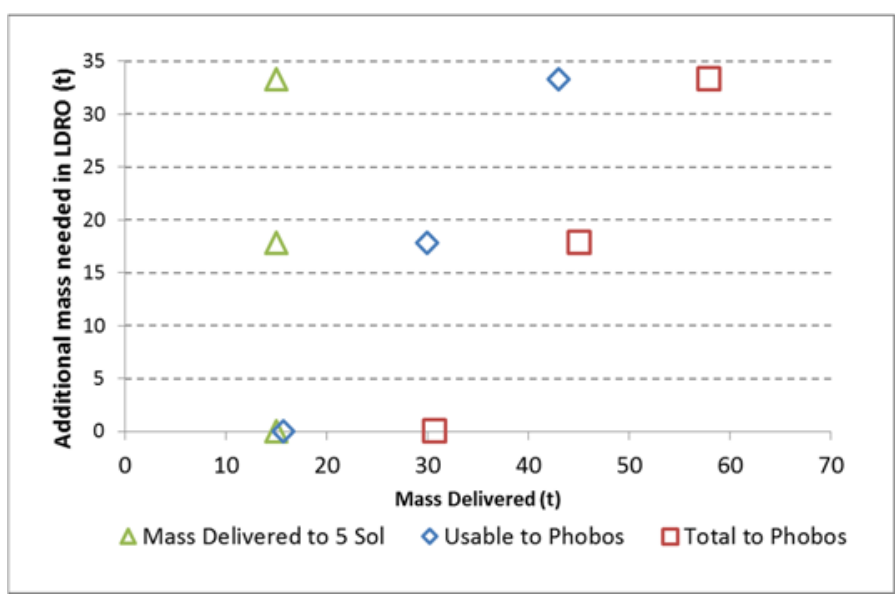

Figure 8. Additional Mass at LDRO vs. Phobos payloads for 2030 Earth departure

\section{Mars Cargo Delivery with HPS reuse}

Mars cargo delivery missions were assessed specifically for two conceptual lander sizes $59 \mathrm{t}$ and $43 \mathrm{t}$, but can also be used for delivery of additional payloads to Phobos for extended missions. The initial cargo mission begins in a manner similar to the Phobos cargo delivery, except once near Mars the cargo to be delivered is released and it captures itself while the HPS does a Mars gravity assist and deadheads back to Earth for recapture (Figure 9). The long duration of these 


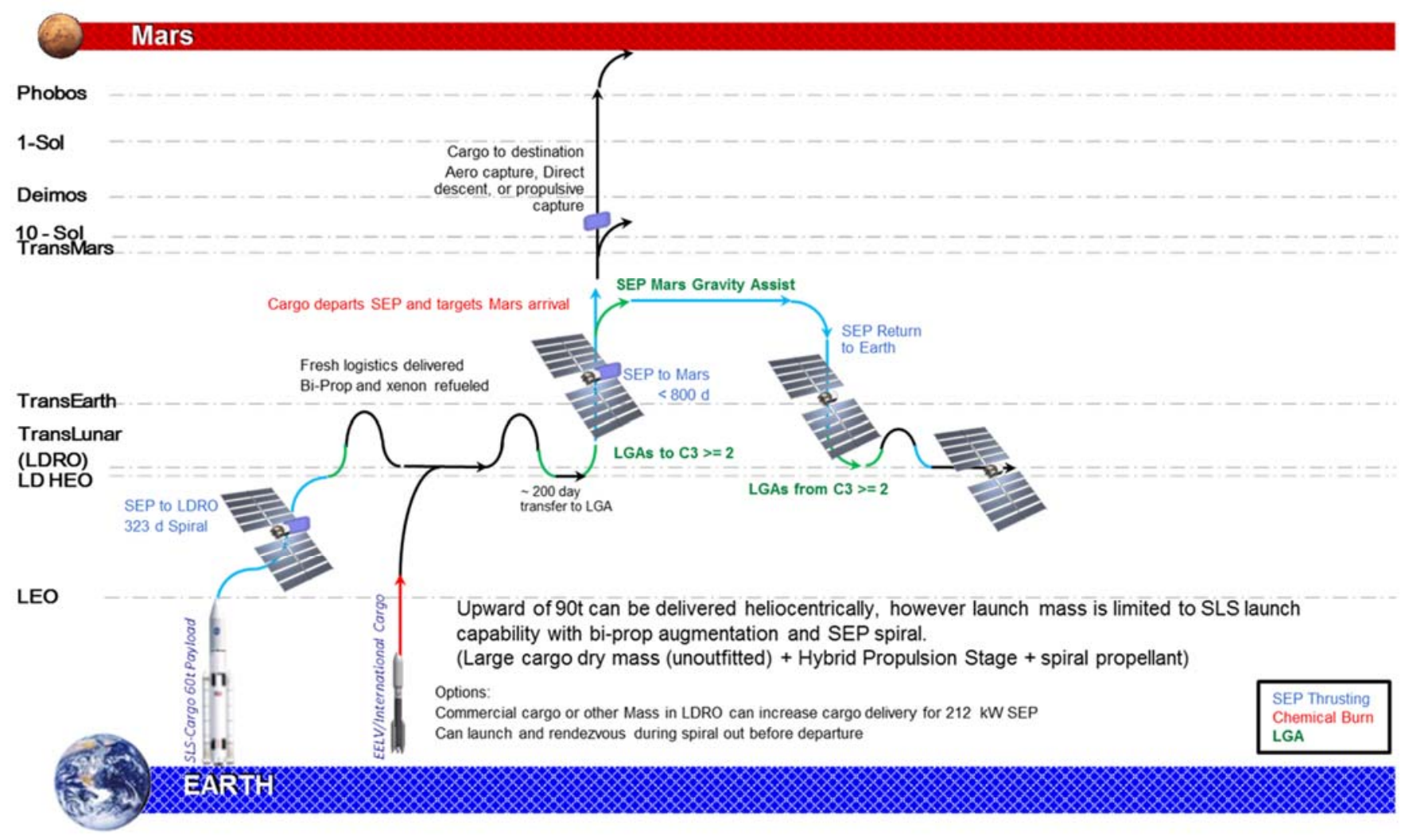

Figure 9: Mars Cargo delivery with HPS reuse

cargo trajectories is a product of using fuel optimal trajectories that are 1.5 revolutions around the sun outbound and inbound. Faster transits in the class of the crew mission are possible as well and are currently being studied. The duration of these trajectories is so long that after capture into the LDHEO upon arrival back at Earth there are only a few orbits prior to departure for refueling and rendezvous with the next payload (lander) that is launched to LDHEO by the SLS. If a cadence of refuel, inspection and cargo rendezvous is achieved then each cargo HPS can be reused to support a cadence of 1 Mars surface mission every other opportunity. In other words, only a few HPS need be operating (3 - 4) and re-used to support multiple Mars surface missions. A replacement strategy for HPS is needed so that all the vehicles aren't replaced at the same time.

\section{Sensitivity Analyses}

Through these analyses, the hybrid architecture is shown to be feasible and to exhibit several significant potential advantages relative to existing Mars architectures. It is important to understand the sensitivity of the concept to varied mission parameters.

\section{Spaceship Mass Sensitivity}

In order for the hybrid architecture to utilize a pre-integrated spaceship, the inert mass of the spaceship must be small enough that a single SLS launch can place the craft on its way to a LDRO. This study initially assumed the maximum inert spaceship mass in a LDRO prior to Earth departure is 65 tons; 75 tons is launched to an elliptical orbit by a SLS 2, and 10 tons of SEP fuel (Xenon) is used to spiral the spaceship to the LDRO. By the end of the study the assumed SLS 2 launch mass to a similar elliptical orbit was only 63 tons with 55 tons arriving in the LDRO. As long as the SLS 2 can deliver the inert vehicle with enough fuel to transit to LDRO the architecture closes. Decreasing the inert mass of the integrated stack reduces the amount of refueling and resupplying that must be accomplished by the crew launch and/or commercial cargo launch(es).

Spaceship mass sensitivity is analyzed using the low-thrust trajectory optimization tool MALTO $[15,16]$. Trajectories are generated for the Earth departure opportunities, minimum payload masses, and EP input powers shown in Figure 10. The solar array output power at $1 \mathrm{AU}$ is twice the SEP input power, rounded to the nearest $100 \mathrm{~kW}$, and the spaceship is assumed to consume a constant $20 \mathrm{~kW}$ of the solar array output power. The CP stage is characterized by a specific impulse of 321s, consistent with a hypergolic stage using an AJ10-190 engine (Space Shuttle Orbital Main Engine). The CP stage is sized with a conservative propellant mass fraction (PMF) of 0.8 , which is less than the 0.86 PMF of a Delta II upper stage that includes $6 \mathrm{t}$ of usable propellant. The three payload mass levels represent three possibilities for crew transportation mass assumptions: 32 tons approximates an 


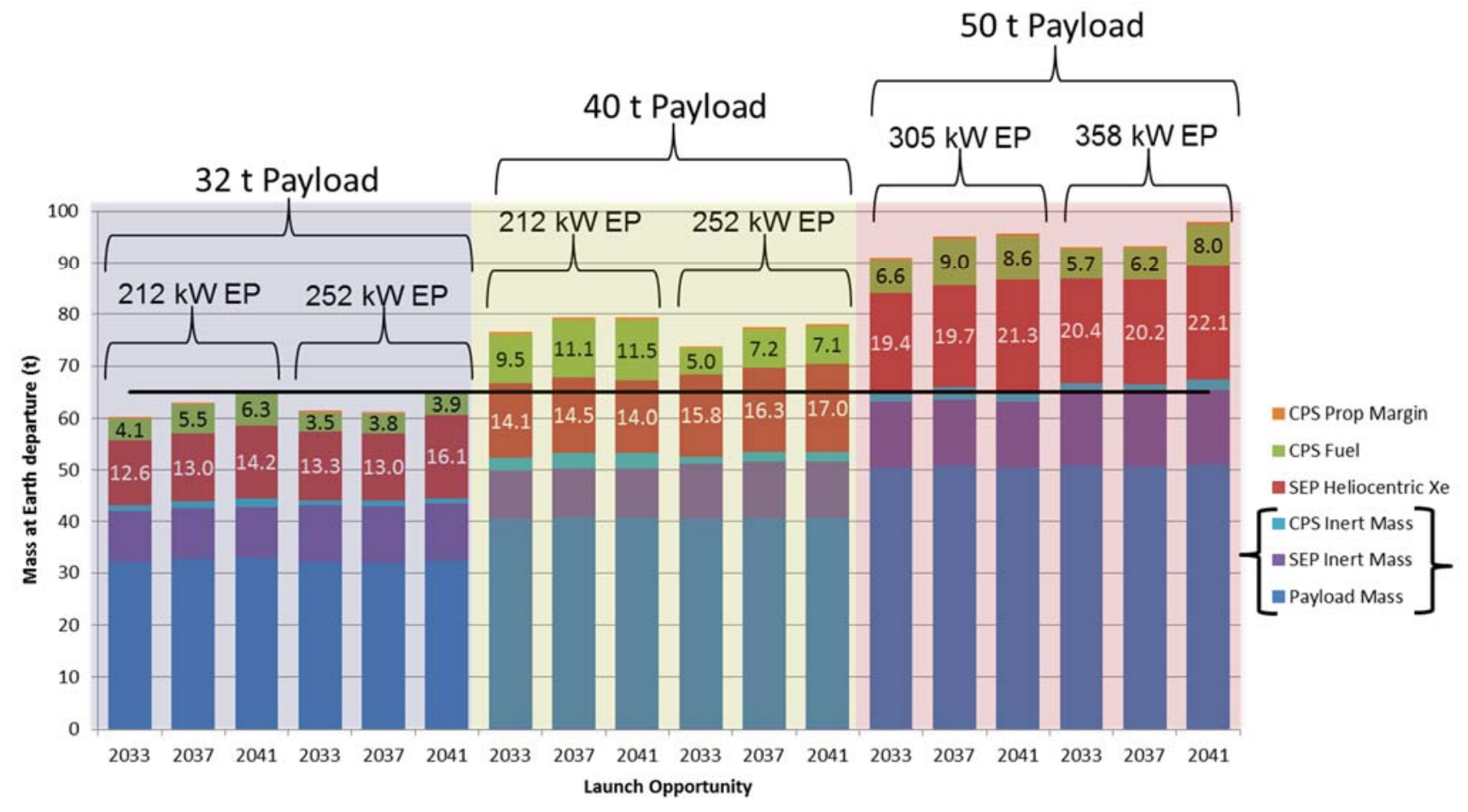

Figure 10: Earth departure mass sensitivity to payload mass, SEP input power, and launch opportunity. Horizontal black line represents maximum mass to LDRO via single SLS launch and SEP spiral.

aggressive estimate of a 4-crew, 600-day Deep Space Habitat (DSH); 40 tons approximates a 4-crew 1100 day DSH; and 50 tons approximates an aggressive DSH and Mars crew taxi vehicle or a heavier DSH.

Figure 10 displays a breakdown of the Earth departure mass for each of the analyzed trajectories. At either $212 \mathrm{~kW}$ or 252 $\mathrm{kW}$ of EP power, 32 tons of payload results in a spaceship wet mass at Earth departure less than 65 tons for 2033 and 2037 Earth departures, and very nearly less than 65 tons for the 2041 Earth departure. Thus, the additional mass that must be brought by the crew or a commercial cargo launch is minimal. For the 40-ton payload case, significant refueling is required, but the inert mass of the spaceship is well below the 65-ton limit for all cases. However, for 50 tons of payload, the inert mass exceeds the 65-ton limit by up to three tons and would require offloading of DSH logistics and spares.

\section{Spaceship Power Reduction Sensitivity}

As the EP input power level decreases, the amount of acceleration the EP delivers to the spaceship decreases, as well. Consequently, the CP stage must compensate for the $\Delta \mathrm{V}$ deficit. From the Tsialkovsky rocket equation, it is well known that the fuel mass required to perform a nearly impulsive chemical burn increases exponentially as $\Delta \mathrm{V}$ increases linearly, a trend seen clearly in Figure 11. The 2033 opportunity is more affected by the loss of SEP input power than the 2037 opportunity over the plotted domain because the optimal flight times for the 2033 opportunity are shorter than for the 2037 opportunity. Thus, the EP has less time to provide incremental acceleration for the 2033 opportunity.
Predictably, as EP input power decreases, the EP fuel requirement decreases, while the $\mathrm{CP}$ fuel requirement increases. However, a second implication of the exponential nature of the rocket equation is the existence of a domain of EP input power over which the overall spaceship mass varies only marginally. For instance, though the spaceship CP fuel requirement increases when the EP input power decreases from $252 \mathrm{~kW}$ to $212 \mathrm{~kW}$, the inert spaceship mass remains nearly constant, and the maximum spaceship wet mass increases by only about 3 tons. Keeping in mind that the 252 $\mathrm{kW}$ EP stage assumes an additional $100 \mathrm{~kW}$ of solar array output power compared to the $212 \mathrm{~kW}$ EP, the additional wet mass may be preferable to the complications introduced by increasing the solar array output by 25 percent.

\section{Elliptical Mars Orbit Effect on Earth Departure Mass}

Other NASA Mars architecture assumes a $250-\mathrm{km}$ periapsis altitude, 1-sol arrival and departure orbit about Mars. Alternatively, the hybrid architecture assumes a 5-sol or10sol orbit with the same periapsis altitude. The larger 10-sol orbit is selected to reduce the $\mathrm{CP}$ stage $\Delta \mathrm{V}$ required for Mars Orbit Insertion (MOI) and trans-Earth injection (TEI). However, Mars orbit selection has several other important impacts on maneuvers within the Mars sphere of influence. First, it is generally unlikely that the Mars approach and departure asymptotes will be aligned such that the Mars arrival orbit will evolve to become the necessary departure orbit at the desired departure time. Therefore, one or more maneuvers are required to align the spaceship for departure. Multiple strategies exist for achieving this task. One single- 


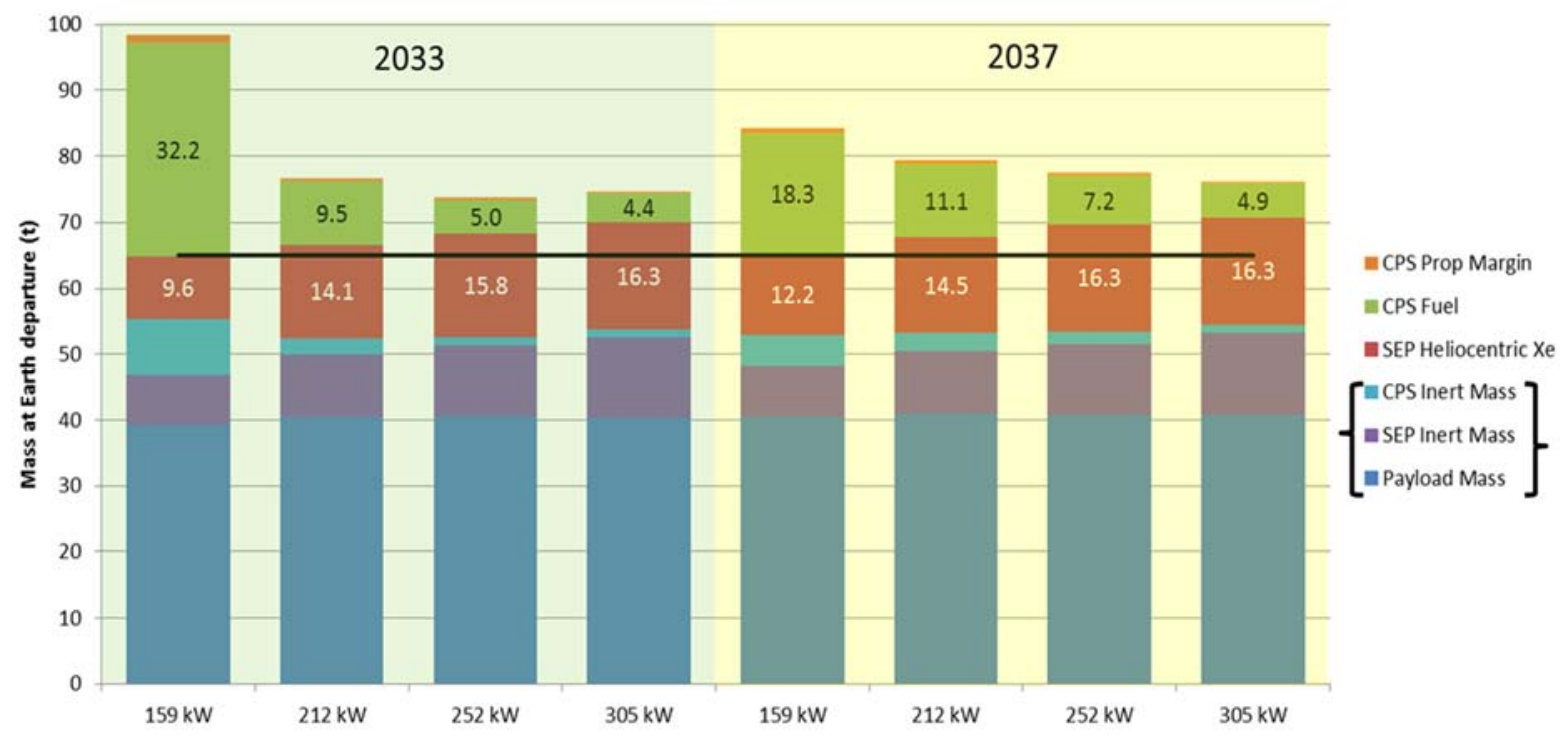

Figure 11: Earth departure mass sensitivity to SEP input power and launch opportunity (40-ton payload). Horizontal black line represents maximum mass to LDRO via single SLS launch and SEP spiral.

maneuver option called the apo-twist uses the natural precession of the arrival orbit (primarily due to the oblateness of Mars) and a plane-change chemical burn at apoapsis [17]. However, initial analysis indicates the apo-twist may not be appropriate for the hybrid architecture. Reasons include (1) the relative lack of precession of the spaceship orbit due to the shorter Mars stay time (and the possibility of the large 10sol orbit) and (2) the relative orientation of the arrival and departure asymptotes for several point designs that have been examined in high fidelity. An alternative approach uses a chemical burn at periapsis to send the spaceship near the edge of the Mars SOI, at which point solar perturbations and a SEP maneuver drive the spaceship to the desired departure orientation. A second chemical burn recaptures the spaceship into an elliptical orbit. The primary fuel costs of the solar- perturbation method lie in reaching and returning from the edge of the Mars SOI. As the size of the arrival/departure elliptical orbit shrinks, the fuel cost grows dramatically, as shown in Figure 12.

The size of the Mars orbit also affects the fuel required to perform crew operations at Mars. For example, a Phobos surface mission requires the spaceship to rendezvous with a crew taxi vehicle, which takes the crew to Phobos orbit and returns the crew from Phobos orbit to the spaceship. An analytical approximation of the $\Delta \mathrm{V}$ required to perform this round trip for a range of spaceship orbit sizes is shown in Figure 5. If only small plane changes are necessary, the 1-sol orbit requires a smaller $\Delta \mathrm{V}$ because of its lower energy. If larger plane changes are necessary, however, a larger

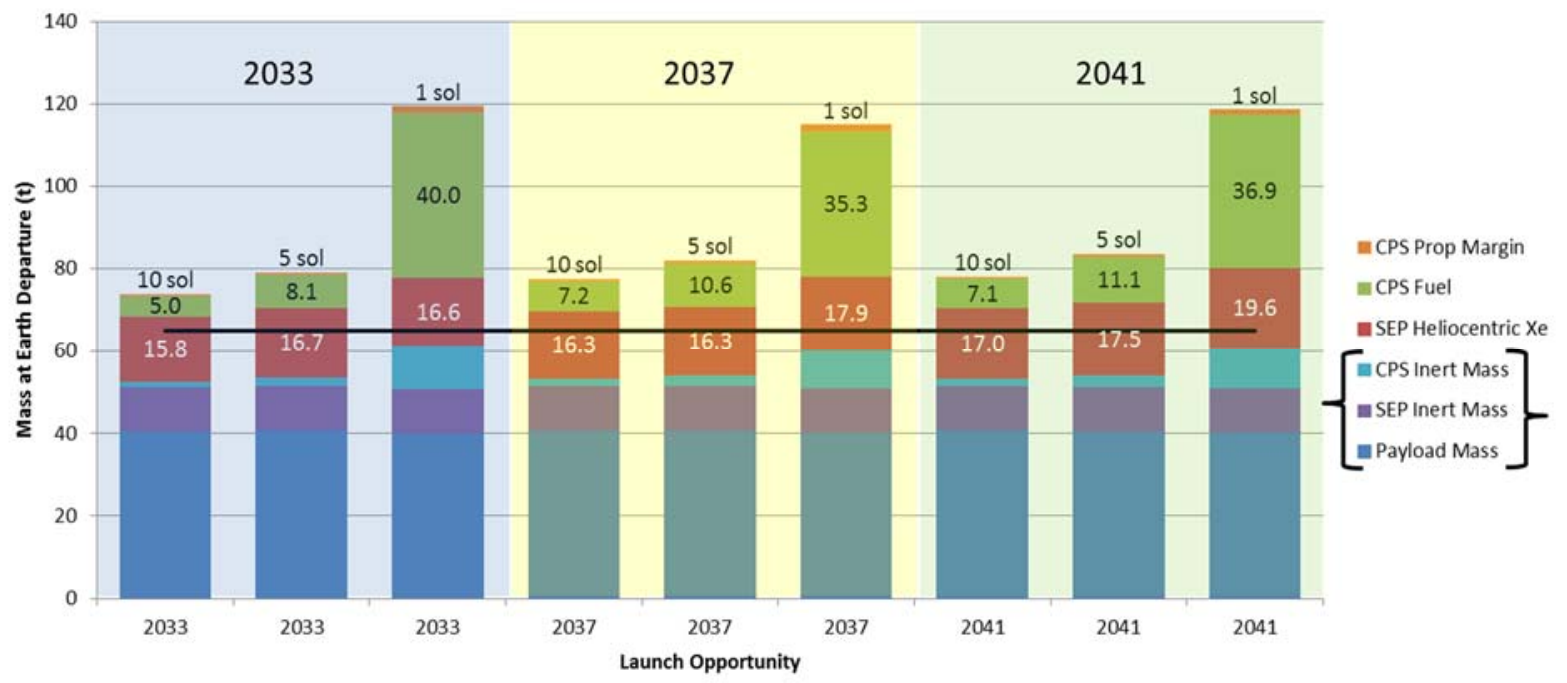

Figure 12: Earth departure mass sensitivity to Mars orbit and launch opportunity (40-ton payload, 252-kW EP). Horizontal black line represents maximum mass to LDRO via single SLS launch and SEP spiral. Solar-perturbation method assumed for spaceship orbit reorientation in Mars SOI. 
spaceship orbit may produce a more efficient set of taxi maneuvers because of the low cost of plane changes far from Mars.

As the taxi payload mass is refined, the time spent by the crew in the taxi must be taken into account, as well. A one-way transfer is likely to be on the order of half a period of the spaceship orbit. Thus, the taxi associated with a 5-sol or 10sol spaceship orbit requires more crew resources than the taxi associated with a 1-sol spaceship orbit.

\section{Evolvability}

In the context of the Evolvable Mars Campaign the Hybrid architecture presents a unique opportunity; it can evolve as NASA's knowledge of long duration exposure to space beyond LEO increases. The high powered SEP can be used to retrieve the resources from near Earth space needed to manufacture structures that serve as both centrifuges and radiation protection. These massive spacecraft could have the same deep space habitat and HPS needed by the Hybrid at their core, but operate in a different manner. If significant additional mass is needed for crew protection the Hybrid can evolve into a ballistic cycler concept, thus never loosing orbital energy once the E-M and M-E trajectories are set. HPS vehicles can serve as reusable cargo carriers to cycler vehicles and the crew taxi developed for Phobos access could be used to perform hyperbolic rendezvous with a modest increase in to use the full CP component of the HPS. This approach would take significantly longer to field for Mars missions, but is clearly possible and likely necessary to maintain crew health for pioneers in the long term if there are no major breakthroughs in in-space transportation technology.

\section{Conclusions}

A new Hybrid transportation architecture is being developed for the Evolvable Mars Campaign that utilizes a single stage reusable spaceship. This "hybrid" approach uses both solar electric and chemical propulsion - a differentiation from current approaches, which use a single propulsion technology for transportation of crew or cargo. The hybrid architecture is shown to have the potential to reduce the risk, complexity, and potentially cost of crewed Mars missions relative to other recent architectures.

A pre-integrated Hybrid spaceship based on existing and indevelopment technologies with performance parameters achievable today is shown to be feasible with significant margin using a single launch under SLS Block 2 capability assumptions. The spaceship may be either fully fueled at launch or refueled and outfitted in a LDRO, depending on payload requirements and SLS capabilities. Additionally, EP power sensitivity analyses demonstrate the feasibility of the hybrid architecture at solar power output levels in the same class as the current split SEP and Chemical EMC architecture.
A 5-sol or 10-sol Mars spaceship orbit - contrasted with other NASA Mars architecture's 1-sol assumption - is shown to be synergistic with the hybrid architecture by shrinking Mars orbit insertion and trans-Earth injection burns. The cost is an increase in the in-plane $\Delta \mathrm{V}$ required of the Mars crew taxi vehicle. However, this disadvantage may be offset if the taxi is required to perform significant out-of-plane maneuvers to reach the target orbit and return to the spaceship. Further, the inert mass of the taxi is significantly smaller than that of the spaceship, which is likely to make minimization of spaceship $\Delta \mathrm{V}$ a higher mission priority than minimization of taxi $\Delta \mathrm{V}$. For Mars surface missions a similar taxi approach can be used to transport the crew between the high elliptical Mars parking orbit and a much lower orbit for rendezvous with landers for descent and ascent.

The potential advantages of the hybrid architecture over other recent Mars transportation architectures include (1) no mission critical rendezvous with return stage in the Mars sphere of influence, (2) the elimination of spaceship integration before Earth departure, (3) an increased potential for habitation and propulsion stage reuse for crew and cargo, (4) a significant reduction in the mass required to be launched from Earth, and (5) evolution paths to mitigate long duration space effects on crew. Thus, the hybrid strategy is an attractive alternative to facilitate crew missions to Mars. It is one of several potential Mars architecture options to be further assessed as part of the Evolvable Mars Campaign.

\section{ACKNOWLEDGMENTS}

This research was carried out at the NASA Langley Research Center and the Jet Propulsion Laboratory, California Institute of Technology (under a contract with NASA), in collaboration with colleagues from NASA's Johnson Space Center, Glenn Research Center, and Marshall Space Flight Center as part of the NASA Evolvable Mars Campaign effort.

\section{REFERENCES}

[1] Crusan, J. (2014). Evolvable Mars Campaign Report to NASA Advisory Council HEO Committee Meeting. http:/www.nasa.gov/sites/default/files/files/20140623Crusan-NAC-Final.pdf

[2] Korsmeyer, D., et al., "A Flexible Path for Human and Robotic Space Exploration” Paper AIAA Space Ops, April 2010, Huntsville, AL.

[3] Parker, J., "TARGETING LOW-ENERGY BALLISTIC LUNAR TRANSFERS", American Astronautical Society (AAS) George H. Born Symposium, Boulder, Colorado. May 13, 2010.

[4] McElrath, T., et al., "Using Gravity Assists in the EarthMoon System as a Gateway to the Solar System", Paper GLEX 2012.05.5.2x12358, May 2012.

[5] Merrill, R. G., et al., Interplanetary Trajectory Design for the Asteroid Robotic Redirect Mission Alternate Approach 
Trade Study. AIAA SPACE 2014 Conference and Exposition, San Diego, CA.

[6] Merrill, R.G., et al., "Cis-Lunar Base Camp", Paper GLEX -2012.05.5.3x12703, May 2012

[7] Merrill, R.G., et al., "An Initial Comparison of Selected Earth Departure Options for Solar Electric Propulsion Missions", Paper IEEE Aerospace Conference 3-10 March 2012, Big Sky Montana

[8] Strange, N., et al., Human Missions to Phobos and Deimos Using Combined Chemical and Solar Electric Propulsion. $4^{\text {th }}$ AIAA/ASME/SAE/ASEE Joint Propulsion Conference \& Exhibit 31 July - 03 August 2011, San Diego, CA.

[9] Strange, N.J., et al., "Solar Electric Propulsion for a Flexible Path of Human Exploration," Paper IAC-10A5.2.4, Sep. 2010

[10] Mercer, C.R., Oleson, S.R., and Drake, B. “A Combined Solar Electric and Storable Chemical Propulsion Vehicle for Piloted Mars Missions", Paper AIAA SPACE 2013 Conference; 10-12 Sep. 2013; San Diego, CA; United States

[11] Kyle, Ed, "NASA's Space Launch System" Space Launch Report - Space Launch System Data Sheet, http://www.spacelaunchreport.com/sls0.html 5/27/2014

[12] Toups, L., et al, "Design and Parametric Sizing of Deep Space Habitats Supporting NASA's Human Spaceflight Architecture Team", Paper GLEX - 2012.05.3.5.x12280

[13] Troutman, P., "The Evolvable Mars Campaign - The Moons of Mars as a Destination", Presentation $11^{\text {th }}$ Meeting of the NASA Small Bodies Assessment Group July 29-31; 2014 Washington, DC

[14] Stich, S., "Asteroid Redirect Mission Crewed Mission (ARCM) Concept Study", Charts http://www.nasa.gov/sites/default/files/files/AsteroidCrewed-Mission-Stich-TAGGED2.pdf

[15] Sims, J. A., \& Flanagan, S. N. (1999). Preliminary Design of Low-Thrust Interplanetary Missions. AAS/AIAA Astrodynamics Specialists Conference. Girdwood, AK.

[16] Sims, J. A., Finlayson, P. A., Rinderle, E. A., Vivrina, M. A., \& Kowalkowski, T. D. (2006). Implementation of a Low-Thrust Trajectory Optimization Algorithm for Preliminary Design. AIAA/AAS Astrodynamics Specialist Conference.

[17] Landau, D. F., Longuski, J. M., \& Penzo, P. A. (2005). Method for Parking-Orbit Reorientation for Human Missions to Mars. Journal of Spacecraft and Rockets, 42(3), 517-522. doi:10.2514/1.7042

\section{BIOGRAPHY}

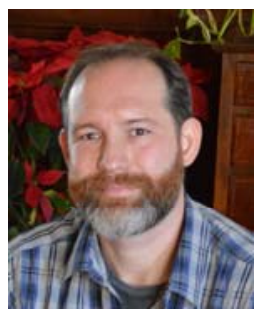

Raymond G Merrill (Gabe) received a B.S. in Aerospace Engineering from North Carolina State University in 2001 and has been at NASA Langley Research Center ever since. He is currently supporting the integrated Mars transportation team and serving and technical deputy at Langley for the Human exploration Architecture Team (HAT) as well as supporting the mission design team for the Asteroid Redirect Robotic Mission Recently he was deputy for the Asteroid Robotic Redirect Mission Alternative Approach Trade Study Option B team. He leads the NASA Langley Space Mission Analysis Branch Mission Design Team and has supported various NASA exploration studies including Launch Vehicle, Lunar, NEA, and Mars missions over the past 14 years.

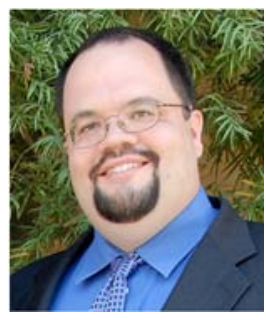

Nathan Strange has worked at NASA's Jet Propulsion Laboratory (JPL) since 2000, where he is currently a systems engineer for mission formulation in the Mission Concepts Section. He holds a M.S. in Aeronautics \& Astronautics from Purdue University and is pursuing a Ph.D. degree with Professor Jim Longuski in Astrodynamics and Space Applications. Nathan is the Mission Design lead for the Asteroid Redirection Robotic Mission concept which aims to retrieve a small asteroid or boulder from a larger asteroid and place it in a stable orbit of the Moon where it can be visited by astronauts as a precursor to human interplanetary missions.

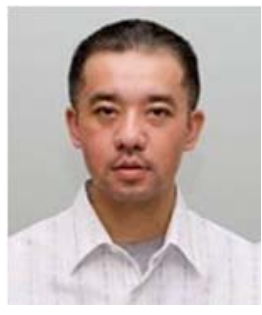

Min $Q \boldsymbol{u}$ received a B.S. in Computational Mathematics from Peking University in 1990, a M.S in Applied Mathematics from Purdue University in 1993, and a Ph.D. in Applied Mathematics from Purdue University in 1997. He has been with NASA Langley for more than 10 years. He is currently supporting the mission analysis and design studies for NASA's Asteroid Redirect Mission (ARM) and Human spaceflight Analysis Team (HAT). Prior to ARM and HAT, he supported various NASA projects and studies including lunar trajectories for Constellation, launch vehicle design and sizing for the Next Generation Launch Technologies (NGLT), tool integration for Advanced Engineering Environment (AEE), and mass property optimizations for the Orion Abort Flight Test project. 


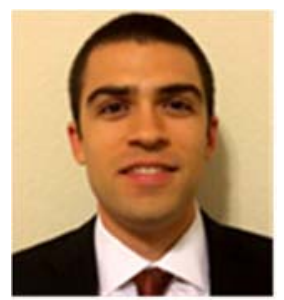

Noble Hatten received a B.S. and M.S. in Aerospace Engineering from the University of Texas at Austin in 2011 and 2012, respectively. He currently works as a graduate research assistant and assistant instructor at UT Austin while pursuing a Ph.D. in Aerospace Engineering. He spent the summers of 2013 and 2014 as an intern in the Space Mission Analysis Branch at NASA Langley Research Center. His research interests include astrodynamic algorithm development and spacecraft trajectory design and optimization. 
